\title{
Structural interaction between bone and implants due to arthroplasty of the first metatarsophalangeal joint
}

\author{
Marco A. Martínez Bocanegra ${ }^{\mathrm{a}}$, Javier Bayod Lopez ${ }^{\mathrm{b}}$, A. Vidal-Lesso ${ }^{\mathrm{c}, *}$, \\ Ricardo Becerro de Bengoa Vallejo ${ }^{\mathrm{d}}$, Raúl Lesso-Arroyo ${ }^{\mathrm{e}}$ \\ a Department of Mechanical Engineering, Universidad de Guanajuato-DICIS, Mexico \\ ${ }^{\mathrm{b}}$ Group of Structural Mechanics and Materials Modeling (GEMM), Aragon Institute of Engineering Research (I3A), Universidad de Zaragoza, Spain \\ ${ }^{\mathrm{c}}$ Department of Mechanical Engineering, Universidad de Guanajuato-DICIS, Comunidad de Palo Blanco, Carretera Salamanca-Valle de Santiago Km 3.5+1.8, \\ C.P. 36880 , Salamanca, Guanajuato, Mexico \\ 'Escuela Universitaria de Enfermería, Fisioterapia y Podología, Universidad Complutense de Madrid, Spain \\ e Department of Mechanical Engineering, Instituto Tecnologico de Celaya, Mexico
}

\section{A R T I C L E I N F O}

\section{Article history:}

Received 14 November 2016

Received in revised form 26 September 2017

Accepted 2 October 2017

Available online $\mathrm{xxx}$

\section{Keywords:}

Metatarsophalangeal joint

Arthroplasty

Finite element

Silicone implants

Biomechanics

\begin{abstract}
A B S T R A C T
Background: Currently, the metatarsophalangeal joint replacement through a restorative arthroplasty, where implants are used, is a viable invasive surgical medical procedure in the treatment of severe cases of osteoarthritis in this joint, better known as hallux rigidus. However, few things are known about the postoperative complications that implants can cause on the joint, like Swanson and Tornier implants. Research in this field can provide a valuable information that would help the specialist surgeon in the decision-making during the selection of the more suitable joint implant in each patient, as well as the redesign of the devices, to make them more efficient, durable and biocompatible with the human body. Methods: The aim of this work is to perform a structural biomechanical analysis of a restorative arthroplasty of the first metatarsophalangeal joint, and to analyze the interaction between bone and medical grade silicone implants. For that, a simulation of a foot with Swanson and Tornier joint implants were performed to evaluate the stress/strain distribution during a critical stage (toe-off).

Results and conclusions: Principal stresses obtained for the first metatarsal with both implants suggest that failure is induced in this bone because, values exceed (up to 136.84\% for Swanson model) the tensile strength reported for phalange trabecular bone, which may be related to osteolysis. Stress and strain values obtained in this work suggest that arthroplasty surgery with Swanson implant is more likely to cause postoperative complications versus Tornier implant.
\end{abstract}

(C) 2017 European Foot and Ankle Society. Published by Elsevier Ltd. All rights reserved.

\section{Introduction}

Osteoarthritis $(\mathrm{OA})$ is the disease characterized by a gradual and progressive destruction of cartilage that covers the surface of joints. It is the most common joint disease worldwide, affecting an estimated $10 \%$ of men and $18 \%$ of women over 60 years of age [1,2]. Osteoarthritis in the first metatarsophalangeal joint, known as hallux rigidus, is the most frequent arthrosis of the foot and ankle, and the second most important condition involving the metatarsophalangeal joint of the big toe in the population, only after hallux valgus [3]. Hallux rigidus affects between 2.5 to $5 \%$ of the

\footnotetext{
* Corresponding author.

E-mail address: agustin.vidal@ugto.mx (A. Vidal-Lesso).
}

population over 50 years [4]; however, it may also occur at earlier ages.

The symptomatology caused by hallux rigidus results in pain, osteophyte appearance and loss of joint mobility, mainly when trying to perform a dorsal flexion movement. The etiology of this pathology is multifactorial, so there is not a specific cause that generates its appearance; however, its occurrence is attributed to factors of origin traumatic, anatomic, structural, metabolic, biomechanical, neuromuscular, postoperative, among others $[5,6]$.

Currently, for the treatment of hallux rigidus there are a variety of conservative and surgical medical procedures; however, surgical treatments are indicated in most cases, so it is indispensable by medical specialists to have an extensive knowledge of the various surgical techniques to recommend the correct treatment for each patient. For the selection of any surgical method, aspects should be 
considered as the grade to the pathology in the patient, age, and physical activity.

In advanced grade of pathology, arthroplasties are used to correct the problem, as shown by Herrera et al. in his proposals of algorithms for the treatment of hallux rigidus $[3,4]$. There are three types of arthroplasties: resection arthroplasty, interposition arthroplasty, and restorative or prosthetic arthroplasty. The present research focuses on a restorative arthroplasty. This invasive surgical procedure involves a prosthesis or joint implant that acts mainly as a dynamic spacer. Which, supports the body weight, restore the joint movement, maintains sesamoid joint, and the length of the metatarsal is conserved [7].

Joint implants made of medical grade silicon are commonly used in restorative arthroplasties, as the Swanson (see Fig. 1A) and Tornier (see Fig. 1B) implants. Several postoperative problems have been reported for the Swanson Silastic ${ }^{\circledR}$ implants. For example: foreign body granulomas and reactive synovitis caused by the material (silicone). In addition, it has been reported that material fractures may occur due to fatigue or some accidental cutting due to sharp instruments during surgery. Also, implant loosening, joint pain, bone erosion, bone resorption and metallosis, due to metal parts of implants with grommets, may appear [7,8].

On the other hand, about the implant designed by Tornier few information is known about its real behavior under working conditions. However, one of the advantages of this implant is the grommets. It is known that the versions of silicone implants with grommets can increase the life of implants [7], but this issue has not been addressed yet and our hypothesis is that both types of silicone implants, with and without grommets have the same biomechanical behavior inside the bones of the first metatarsophalangeal joint. In most cases, the postoperative effects that these prostheses can cause on the joints have not been studied enough. Surgeons pay attention mainly on treating the symptoms caused by the pathology and restoring the function of the joint.

Because of the lack of long-term studies about the postoperative complications that the Swanson and Tornier implant can cause in the metatarsophalangeal joint of the big toe, as well as, their mechanical behavior in working conditions; the generation of knowledge through the use of finite element in this area, with the generation of 3-D models capable of predicting a close response with reality, can be a good information tool for preoperative decision-making of surgeons and podiatrist, at the time of selecting the more appropriate implant according to the case presented. Also, it can be used as a tool to detect the critical areas of the prosthesis with the purpose that, in the future, work can be done on the redesign to make them more efficient, durable and biocompatible with the human body.

Therefore, the aim of this work is to perform a structural biomechanical analysis of the arthroplasty of the first metatarsophalangeal joint to study the interaction between bone and two commercial joint silicone implants; using a finite element musculoskeletal model of a foot. It is important to mention that there are already studies in this field; however, the models used have been limited to 2-D (Lewis et al. [9]) or 3-D (Flavin et al. [10]; Williams et al. [11]) geometries, where the implants have been studied alone or the interaction between them and the joint, using

(A)

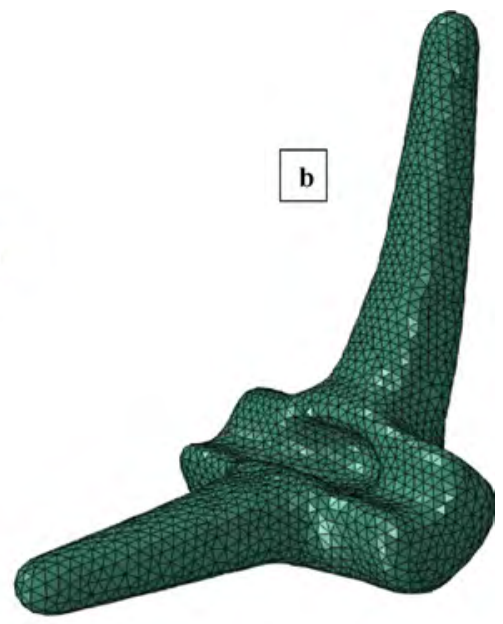

(B)

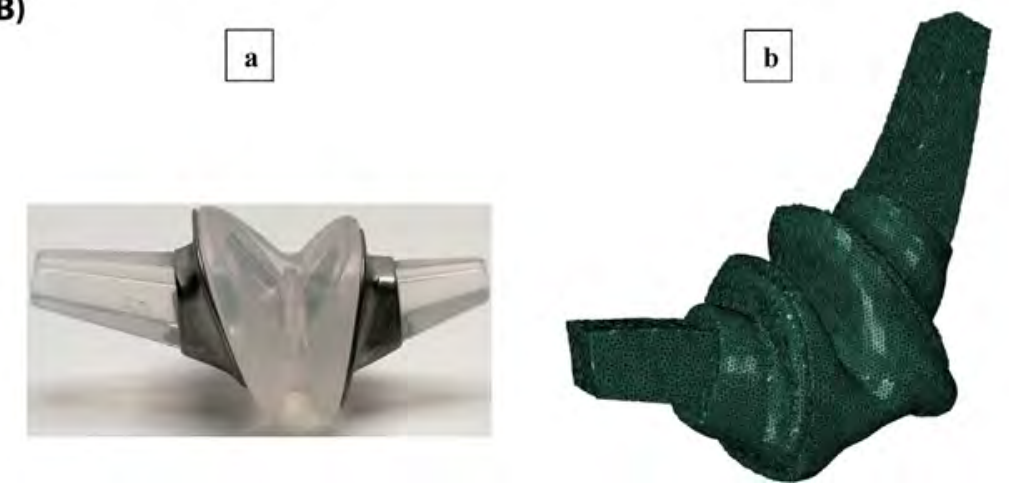

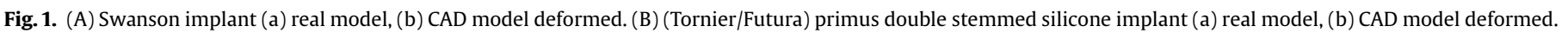


only some muscles. However, in this study the analysis is performed with a whole complete model, achieving a better distribution of the loads between the bones and a better result of the effects that these types of implants can cause in joint patients, as well as, the mechanical behavior of them.

\section{Materials and methods}

\subsection{Generation of the foot model}

The foot model used in this research is a finite element model developed by the Group of Structural Mechanisms and Material Modeling (GEMM), located in the Campus Rio Ebro of the School of Engineering and Architecture of the University of Zaragoza (Spain).

For the generation of the foot model bones, 93 DICOM format Computerized Tomography (CT) scans were taken at every $2 \mathrm{~mm}$ interval from the frontal plane of a 36 year old male's right foot, who presented no malformation or pathology. For that, a software developed by the Advanced Computing Group (GIGA) of the University of Zaragoza was used. The methodology for the creation of the bone volumes was the following: firstly, the CT scans were loaded in the correct order to the software. Afterwards, the contours for each bone were profiled by means of splines with nodes. Finally, the cortical (compact bone or higher density) and trabecular parts (porous bone or Lower density) were segmented. With these, the software automatically obtained the volume of the bones that are part of the foot model. The purpose of modeling the cortical and trabecular parts of each bone was to characterize their different mechanical behavior and to obtain a biomechanical response closer to their real behavior under critical working conditions. The commercial software HARPOON (Harpoon r1.4.5, CEI, Manchester, England) was used to mesh the cortical and trabecular bone. Linear tetrahedra mesh with an average size of about $1 \mathrm{~mm}$ was used. The bones were assembled in a position simulating the stage or event known as "toe-off" in which it is in the "third rocket" on the stance phase [12]. The reason is that, when the joint implant interacts with the bones of the metatarsophalangeal joint, in the foot model, a static critical stage exists, due to in this position the loads are mainly concentrated on the head of the metatarsals and phalanges bones [13].

Soft tissues have been added to the model into this work (cartilages, thin ligaments, long ligaments, plantar ligaments and muscles). Currently, the foot model consists of 26 bones: 14 phalanges (proximal, medial and distal), 5 metatarsals, 3 cuneiforms, the cuboid, navicular, talus and calcaneus. Two sesamoid bones were also included as a unique volume bonded to the first metatarsal. The geometry also includes thin ligaments (561), long ligaments and plantar ligaments (49). Cartilage and eight muscles were considered, among them were included: the extensor hallucis longus with its expansion and capsularis, extensor hallucis brevis, flexor hallucis longus, flexor hallucis brevis, abductor hallucis, adductor hallucis, flexor digitorum longus and flexor digitorum brevis (see Fig. 2). It is important to note that soft tissues do not have a true geometry, it is a structural effect that is considered into this work. The insertion of soft tissues in the model was defined according to anatomy books $[14,15]$.

\subsection{Arthroplasty characterization}

Bones and soft tissues that are part of the first metatarsophalangeal joint (proximal phalanx, metatarsal bone, cartilage, thin ligaments and muscles), were separated from the whole foot model. Thus, ligaments and articular cartilage of the head of the metatarsal bone and base of the proximal phalanx were removed. Two parallel cuts to the frontal plane were done (no angled cuts), one of $2 \mathrm{~mm}$ size in the base of the proximal phalanx and the other one of $3 \mathrm{~mm}$ in the metatarsal head, for the case of the joint with model Swanson implant. For the case of the joint with the model Tornier implant, a similar procedure was done. The amount of extracted bone was removed with the aim of to avoid the change in the big toe length, since the increment or reduction on its length may cause biomechanical imbalances in the foot, leading to the development of future pathologies [16]. Also, it was done to avoid compromising the insertion of the tendons in the proximal phalanx base, which later may cause foot plantar weakness and

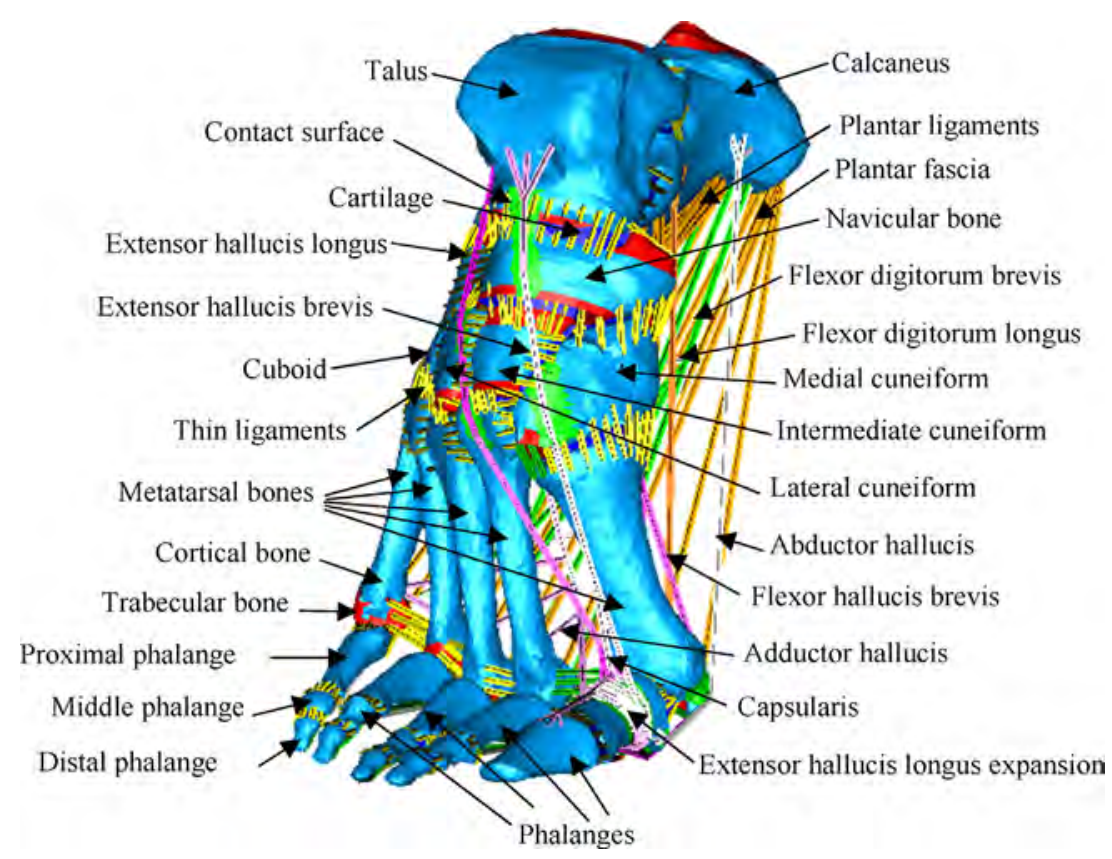

Fig. 2. Tissues considered on the foot model. 


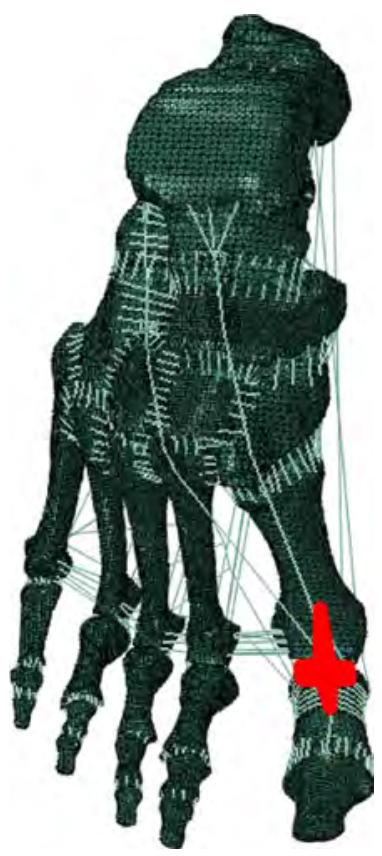

Fig. 3. Characterization of the arthroplasty in the first metatarsophalangeal joint of the foot model.

eventually generates problems in the small joints, like metatarsalgia, claw toe, hammer toe, hallux valgus, etc. [17]. Finally, the amount of extracted bone helps to remove the damaged bone due to the hallux rigidus.

Swanson and Tornier commercial implants considered in this work were scanned to obtain the 3-D models, in the case of the Tornier implant, the stems were shaped with a rectangular geometry because of problems with the scanner. The most notorious difference between them, besides the geometry, were the grommets in the implant Tornier. Metal grommets, when they are implanted, can increase the useful life of the prosthesis. Grommet allows the metal to take the load of the piston type, instead of the bone. The Grommet provides a solid surface against the bone in which the piston movement of the joint is minimized [7]. For each foot model, the implant was deformed until it reached the angle formed by the bones of the metatarsophalangeal joint of the big toe in the "toe-off" stage. Later, the prosthesis was inserted in the joint (see Fig. 3), simulating the restorative arthroplasty. For the analysis in the foot models, the stress state due to implants pre-flexion to achieve the toe-off stage was not considered into this work. Furthermore, it is important to know that the prosthesis position inside of the joint was supervised by an orthopedic surgeon and podiatrist, PhD, Ricardo Becerro de Bengoa Vallejo.

Once the implant was located inside the joint, it was meshed again with tetrahedral elements; for the case of the joint with the Swanson implant (bones and prosthesis), an element size of approximately $1 \mathrm{~mm}$ was used. For the case of the articulation with the Tornier implant, an element size of $1 \mathrm{~mm}$ was used for the bones and a smaller size for the prosthesis was considered to avoid numerical singularities and lost geometry due to mesh.

The new metatarsophalangeal joints, with their respective implant, were positioned in the remaining foot model and soft tissues (articular cartilage, thin ligaments, plantar foot ligaments, muscles), and contact surfaces were modeled in the whole foot model. Tetrahedral elements of $1 \mathrm{~mm}$ of size were used to model the articular cartilage. Muscles and ligaments (see Fig. 4) were modeled with non-compression beam elements, and only those muscles with a considerable influence on toes behavior and specifically those related to big toe [14,15]. A frictionless contact, between muscle and bones, was defined through contact surfaces (shell elements) created where an interaction between these elements existed. The final foot model with arthroplasty of the first metatarsophalangeal joint with Swanson implant was formed by 896,675 elements and 141,946 nodes; while the final model with the Tornier implant was formed by $1,535,877$ elements and 282,330 nodes.

\subsection{Mechanical characterization of the tissues of the musculoskeletal model}

All bones and soft tissues of the foot model were considered as homogeneous, isotropic and linearly elastic deformable

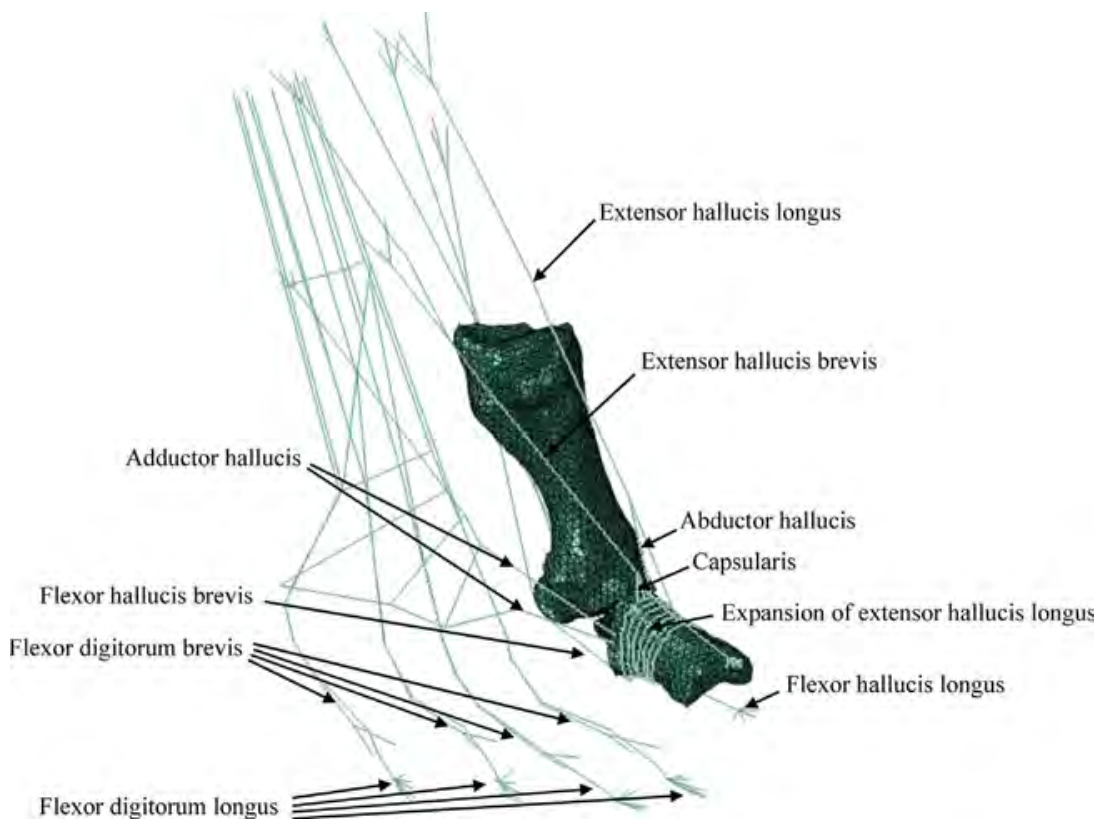

Fig. 4. Muscles. 
Table 1

Mechanical properties of bones, soft tissues and implants.

\begin{tabular}{lcc}
\hline Tissues & Young's modulus $(\mathrm{MPa})$ & Poisson's ratio $(v)$ \\
\hline Cortical bone & 17,000 & 0.3 \\
Trabecular bone & 700 & 0.3 \\
Muscles & 450 & 0.3 \\
Cartilage & 10 & 0.4 \\
Ligaments & 260 & 0.3 \\
Plantar ligaments & 350 & 0.3 \\
Implant (silicone) & 15 & 0.48 \\
Titanium (grade 5) & 113,800 & 0.342 \\
\hline
\end{tabular}

bodies. Young's modulus and the Poisson coefficient for the cortical bone were established as $17,000 \mathrm{MPa}$ and 0.3 , respectively, while for the trabecular bone Young's modulus of $700 \mathrm{MPa}$ and a Poisson coefficient of 0.3 were considered. Young's modulus assigned to cartilage was of $10 \mathrm{MPa}$, and a Poisson coefficient of 0.4 was used. Ligaments were modeled by non-compression elements considering two groups: the first, for superficial long plantar ligament and fascia ligaments with Young's modulus of $350 \mathrm{MPa}$, Poisson ratio of 0.3 and a crosssection of $290.7 \mathrm{~mm}^{2}$. And the second, that corresponds to the rest of ligaments with Young's modulus of $260 \mathrm{MPa}$, Poisson ratio of 0.3 , and a cross-section of $18.4 \mathrm{~mm}^{2}$. In addition, muscles were assessed with Young's modulus of $450 \mathrm{MPa}$, Poisson ratio of 0.3 , and a cross-section of $12.5 \mathrm{~mm}^{2}$. Respect to the Young's modulus for implants (medical grade silicone) was $15 \mathrm{MPa}$ and a Poisson coefficient of 0.48 . Finally, for the Titanium (grade 5) Young's modulus and Poisson coefficient were considered of 113800 and 0.342 , respectively [18-22]. The mechanical properties assigned to the tissues and implants are shown in Table 1.

It is well known that the material's behavior of some tissues is not linear elastic, isotropic and homogeneous. However, as small strains and displacements are considered, the overall behavior of the foot model is close to reality.

\subsection{Boundary conditions}

The magnitude of the loads applied to the foot model was obtained from literature data where the effects of a person of $60 \mathrm{~kg}$ are considered. The magnitude and direction of the load that a person with this body weight apply on the talus in the "toe-off" stage are of $1805 \mathrm{~N}$ to $20^{\circ}$ clockwise (see Fig. 5) in the sagittal plane $[18,23]$. This load was applied throughout a group of 361 nodes located in the zones where the tibia and fibula contact the talus.

Pre-load magnitudes on the muscles due to toe-off stage were obtained in relation to the $2 \%$ of strain of these tissues, equivalent to $22 \mathrm{~N}$ applied to the digitorum longus and digitorum brevis flexor of the $2^{\text {nd }}, 3^{\text {rd }}, 4^{\text {th }}$ and $5^{\text {th }}$ toe and $11 \mathrm{~N}$ was applied in the hallucis

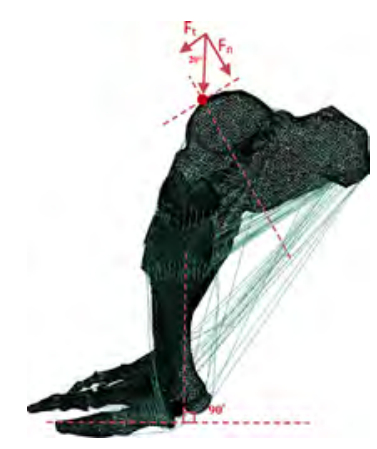

Fig. 5. Load on the model. longus extensor, hallucis brevis extensor, abductor hallucis and adductor hallucis $[18,23]$.

As for the restrictions of displacement in the foot model, displacements of proximal and distal phalanges of the five toes were constrained. All degrees of freedom were constrained at the base and head of the $1^{\text {st }}$ proximal phalanx, as well as the base of the $2^{\text {nd }}$ and $3^{\text {rd }}$ proximal phalange. For the distal phalanges, rotation and translation in Z-direction were constrained at the head of the $2^{\text {nd }}, 3^{\text {rd }}, 4^{\text {th }}$, and $5^{\text {th }}$ phalanges. Finally, all rotations and translations in the insertion area of the Achilles tendon on calcaneus were constrained to keep the position of the model.

\section{Results}

The magnitude of maximum principal stress for first metatarsal bone with Swanson implant on trabecular bone, where the proximal implant stem contacts the tissue, was of $1.34 \mathrm{MPa}$ (see Fig. 6a). Respect the maximum principal stress to the proximal phalange, in the same model, on the trabecular bone where the distal implant stem makes contact was 7.25 MPa (see Fig. 6b).

Moreover, maximum principal stresses obtained for trabecular and cortical metatarsal bone with Tornier implant model, where the grommet of the proximal stem of the implant contacts the tissues, were $1.205 \mathrm{MPa}$ and $18.12 \mathrm{MPa}$, respectively. For the proximal phalanx, the maximum principal stress value was of 5.027 MPa, this value was obtained from the trabecular bone in the area where the grommet of the distal stem of the implant makes contact with the tissue.

The values of the maximum principal stress reported in this work are average stress values, taken specifically of the outline of the cavity (medullary canal) of areas of interaction between implants and bones of the joint of the big toe. Discarding those singularities (peaks of stress) that were obtained by limitations in the mesh of the model.

Maximum principal stresses for both implants were also obtained. Swanson implant had a stress value of $13.25 \mathrm{MPa}$, which was in the proximal stem (see Fig. 7a). Moreover, Tornier implant presented a stress value of $8.52 \mathrm{MPa}$, it was obtained in the hinge area (see Fig. 7b). The grommets of the Tornier implant had stress values of $28.67 \mathrm{MPa}$ at the distal stem, and $71.52 \mathrm{MPa}$ at the proximal stem.

Maximum principal stresses of the simulations for arthroplasty models with Swanson and Tornier implants were compared with the stress in a model without arthroplasty reported by Morales et al. [21].

In Table 2, the maximum principal stresses for the three models including the model without arthroplasty are shown. It is important to note that the values presented in this table are stresses obtained for bones in contact with the implants. Thus, cortical bone of metatarsal and proximal phalange is not in contact with Swanson implant, as well as, cortical bone of proximal phalange for the Tornier implant is not in contact. These values are not reported.

For a better analysis and understanding of the biomechanical behavior of the interaction of the implants with the bones of the joint, the maximum principal maximal strains were obtained on the outline of the cavity (medullary canal) of areas of interaction between implants and bones too. In Table 3 the maximum principal strains are showed which are obtained in the two models with arthroplasty and in the model without it.

Maximum principal strains for trabecular bone were compared between the models with Swanson implant respect Tonier implant, finding differences in the results up to $85 \%$ for the proximal phalange bone and up to $65 \%$ for metatarsal bone. Now, if we made a comparison of the maximum principal strains for trabecular bone between the model without arthroplasty versus 

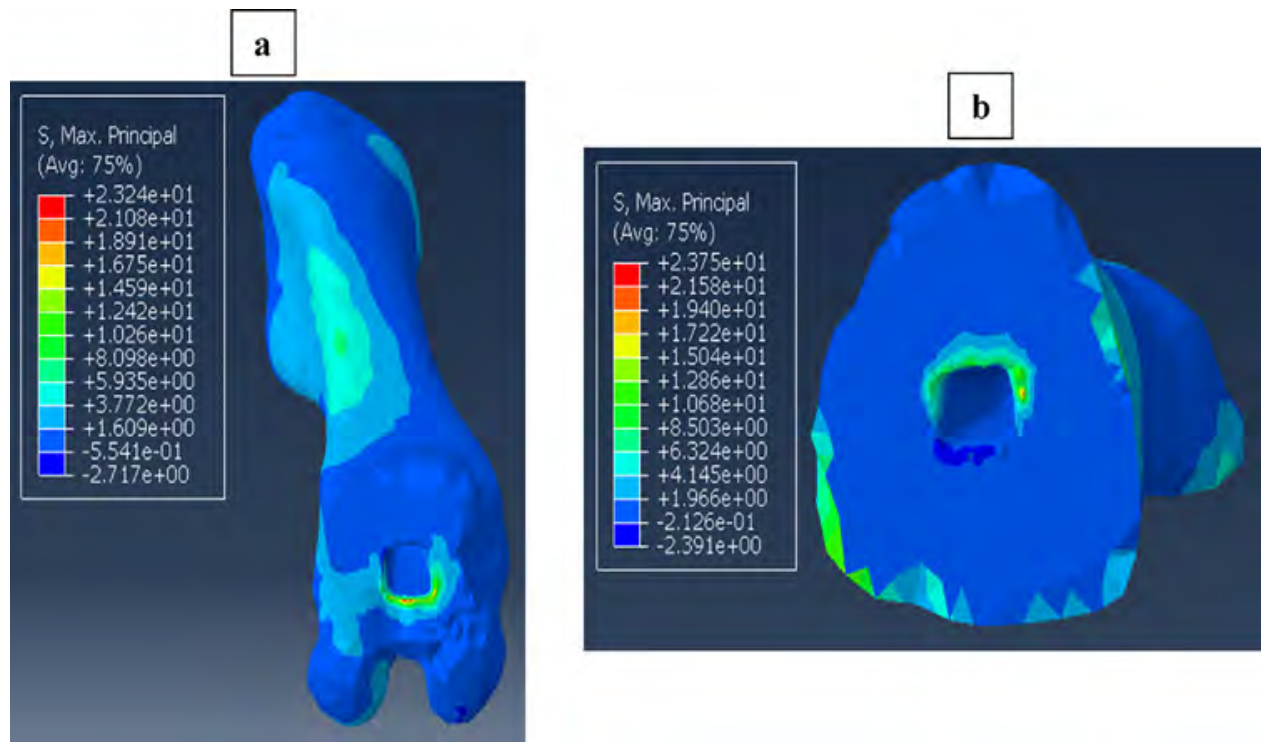

Fig. 6. Maximum principal stress for the model with implant Swanson (a) metatarsal bone, (b) proximal phalanx.
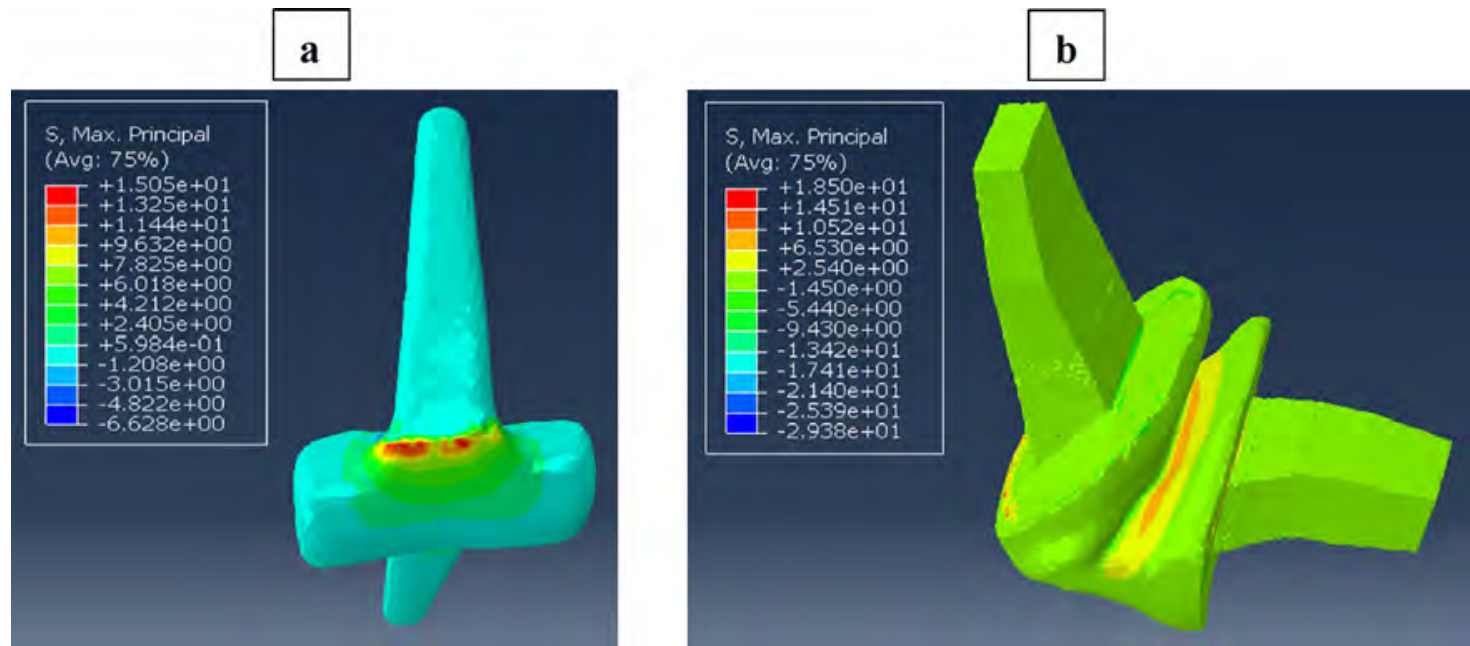

Fig. 7. Maximum principal stresses for implants (a) Swanson implant, (b) Tornier/Futura implant without grommets.

the models with Swanson and Tornier implants, we found that Swanson model presented the largest differences, up to $64 \%$ for the first metatarsal bone and more than $100 \%$ for the proximal phalange bone. On the other hand, for the Tornier implant, a reduction in the maximum principal strains of $0.5 \%$, for the first

Table 2

Maximum principal stress.

\begin{tabular}{|c|c|c|c|}
\hline \multirow[t]{2}{*}{ Model } & \multirow[t]{2}{*}{ Bones } & \multicolumn{2}{|c|}{ Maximum principal stress (MPa) } \\
\hline & & Trabecular bone & Cortical bone \\
\hline \multirow{2}{*}{ Implant (Swanson) } & $1^{\text {st }}$ metatarsal & 1.34 & - \\
\hline & Proximal phalange & 7.25 & - \\
\hline \multirow[t]{2}{*}{ Implant (Tornier) } & $1^{\text {st }}$ metatarso & 1.20 & 18.12 \\
\hline & Proximal phalange & 5.02 & - \\
\hline \multirow[t]{2}{*}{ Without arthroplasty } & $1^{\text {st }}$ metatarsal & 0.97 & 12.08 \\
\hline & Proximal phalange & 3.43 & 25.85 \\
\hline
\end{tabular}

metatarsus was obtained, while an increase of more than $100 \%$ for the proximal phalanx was found.

The maximum values obtained into this work for principal strains in the Swanson and Tornier implant were 0.45 and 0.42 , respectively. Magnitudes of these values are shown in Table 4.
Table 3

Maximum principal strains for bones.

\begin{tabular}{llll}
\hline Model & Bones & \multicolumn{2}{l}{ Maximum principal strains $(\mu \varepsilon)$} \\
\cline { 3 - 4 } & & Trabecular bone & Cortical bone \\
\hline Implant (Swanson) & $1^{\text {st }}$ Pretatarsal & 1961 & - \\
& Proximal phalange & 16960 & - \\
Implant (Tornier) & $1^{\text {st }}$ metatarso & 1186 & 909 \\
& Proximal phalange & 9163 & - \\
Without artroplasty & $1^{\text {st }}$ metatarsal & 1192 & 1220 \\
& Proximal phalange & 2128 & 2650 \\
\hline
\end{tabular}


Table 4

Maximum principal strains for implants.

\begin{tabular}{ll}
\hline Implant & Maximum principal strains $(\mathrm{mm} / \mathrm{mm})$ \\
\hline Swanson & 0.45 \\
Tornier & 0.42 \\
\hline
\end{tabular}

\section{Discussion and conclusions}

Values of maximum principal stresses reported in this work for the trabecular bone of the first metatarsus for Swanson and Tornier implants are below of the failure value (5.3 MPa) reported for trabecular bone by Shin et al. [24]. This suggests that the interaction between implant and trabecular bone for both models does not induce any failure (bone fracture) from the mechanical point of view.

Values obtained for maximum principal stresses for trabecular bone corresponding to proximal phalange for the model with Swanson and Tornier implant showed that, for the model with Swanson implant, these values exceed $136.84 \%$ the tensile strength reported for this tissue [24]. This leads to failure, interpreted as a fracture of the trabecular bone, causing osteolysis (erosion) [25-28]. In addition, stresses obtained for trabecular bone of the proximal phalange with the Tornier implant showed that these values are close to tensile strength (up to 94.84\%) of trabecular bone reported by Shin et al. [24], so premature failure of this tissue may be expected with similar effect as we mention above.

Maximum principal stresses obtained for cortical bones with Tornier implant showed a value of $18.12 \mathrm{MPa}$, this value is below the value reported by Shin et al. [24] for this tissue (175 MPa), this means that cortical bone is not damage. However, this value according to Zeng et al. [29] may damage the periosteum.

On the other hand, some strains values showed in Table 3 for Swanson and Tornier models are above the values reported by Trabersi et al. [30] for microcracks. This means that strains values obtained in bones into this work, lead to microcracks (bone failure) in the trabecular bone of proximal phalanges for both models.

Maximum principal stress obtained for implants was $13.25 \mathrm{MPa}$ for Swanson and $8.52 \mathrm{MPa}$ for Tornier implant, thus, the stress generated in both implants is above (169.87\% and 109.23\%) the tensile strength reported for this material (7.8 MPa) [22], this suggests that material failure leads to early implant fracture under simulated working conditions. These results agree with clinical data and correspond with a failure grade 1 , according to with the criteria of breakage and deformation of the implants studied by Kanzaki et al. [31] and Granberry et al. [32]. They classified as grade 0 (no evidence of deformation or fracture), grade 1 (slight deformation or fracture of the stem or the hinge), and grade 2 (complete deformation of the implant).

Several studies have been developed for the Swanson implant. Among them are the works by Lewis et al. [9] and Williams et al. [11]. They realized an analysis through the finite element method of Swanson implant, where models with and without grommets were studied. They simulated different working conditions, trying to establish an environment of work close to reality, to predict the structural behavior of the implant. The critical stresses reported by Lewis et al. were $0.9,1$ and $0.8 \mathrm{MPa}$ for the maximum principal, maximum principal shear and equivalent stress, respectively. The von Mises critical stress reported by Williams et al. at $90^{\circ}$ was $0.78 \mathrm{MPa}$. These results compared to those obtained in the models characterized with the arthroplasty are very low; however, it must be considered that the analysis done in these investigations was under different working conditions than those that were taken for this analysis. However, in the analysis by Williams et al., a similarity can be seen in the concentration area of maximum stress. It was presented at the bottom where the proximal stem joins with the hinge, area where this type of implant usually fails [33]. Stresses and strain reported in this work (see Tables 2-4) suggest that arthroplasty surgery with Swanson implant is more likely to occur complications [7]. The advantage of the grommets in the Tornier implant reflects a better behavior under load conditions, where the concentration of maximum stresses, instead of being located between the union of the stems with the hinge, it is centered in the hinge, whose function is to allow the free movement of the joint. Thus, in this area, the concentration of stress does not have a negative influence, since it is designed for it.

The strength of this study lies in the use of a complete finite element model for the analysis of the interaction of the implants with the bones of metatarsophalangeal joint of the big toe.

Some limitations are presented in the model of this work, one of them was found with the generation of a coarse mesh and some skew elements presented in some areas of the joint and implants; also, some singularities were found because of the skew mesh. We consider that a more real soft tissue geometry and an appropriate mechanical characterization of each tissue of the musculoskeletal model may be included in a future work. In addition, the pre-stress in the implants due to the deformation suffered by the analysis stage of the foot model was not considered into this work, these pre-stress effects may be calculated to be included into the future work of this study.

In summary, both the Swanson and Tornier implants produce failure on the trabecular bone of the proximal phalanx, as well as on the trabecular bone of the phalanx. Also, it was obtained that the micro-strains reported in the phalanges of both models create micro-fissures causing osteolysis and damage in the periosteum.

On the other hand, it was observed that both implants fail under conditions of analysis established.

Finally, with the analysis and interpretation of results, it is concluded that the Tornier implant is a better option in terms of strength and durability in this type of surgery compared to the Swanson implant. Through this work, it could be seen that the use of finite element models to simulate several phenomena in the field of biomechanics has become a powerful predictive tool. In healthcare, this type of model can help doctors in the evaluation of pathologies, surgical procedures, selection of prosthesis, modeling the behavior of some cell, tissue, organ or physiological process, helping to predict, prevent or reduce future complications affecting the quality of human being.

\section{Conflict of interest}

The authors declare no conflict of interest.

\section{Acknowledgment}

We are grateful to the project DPI2016-77016-R of the Ministry of Economy and Competitiveness of the Spanish government.

\section{References}

[1] Glyn Jones S, Palmer A, Agricola R, Price A, Vincent T, Weinans H, et al. Osteoarthritis. Lancet 2015;386(9991):376-87, doi:http://dx.doi.org/10.1016/ S0140-6736(14)60802-3.

[2] Johnson VL, Hunter DJ. The epidemiology of osteoarthritis. Best Pract Res Clin Rheumatol 2014;28(1):5-15, doi:http://dx.doi.org/10.1016/j.berh.2014.01.004.

[3] Herrera Perez M, Pais Brito JL, Valderrabano V, Cortés Garcia P, Déniz Rodríguez B, Ayala RA. Propuesta de algoritmo terapéutico para hallux rigidus. Acta Ortop Mex 2014;28(4):253-7. http://www.medigraphic.com/pdfs/ortope/or-2014/ or144j.pdf.

[4] Herrera Perez M, Andarcia Bañuelos C, de Bergua Domingo J, Paul J, Barg A, Valderrabano V. Propuesta de algoritmo global de tratamiento del hallux rigidus segñn la medicina basada en evidencia. Rev Esp Cir Ortop Traumatol 2014;58(6):377-86, doi:http://dx.doi.org/10.1016/j.recot.2014.05.004. 
[5] Asunción Márquez J, Martín Oliva X. Hallux rógidus: etiologóa, diagnástico, clasificacián y tratamiento. Rev Esp Cir Ortop Traumatol 2010;54(5):321-8, doi:http://dx.doi.org/10.1016/j.recot.2010.05.005.

[6] Vanore John V, Christensen Jeffrey C, Kravitz Steven R, Schuberth John M, Thomas James L, Weil Lowell Scott, et al. Diagnosis and treatment of first metatarsophalangeal joint disorders. Section 2: hallux rigidus. J Foot Ankle Surg 2003;42(3):124-36, doi:http://dx.doi.org/10.1053/jfas.2003.50037.

[7] Turner Warren A, Merriman Linda M. Habilidades clínicas para el tratamiento del pie. Segunda edición España, S.A: Elsevier; 2007.

[8] Esway Jan Eric, Conti Stephen F. Joint replacement in the hallux metatarsophalangeal joint. Foot Ankle Clin N Am 2005;10:97-115, doi:http://dx.doi.org/ 10.1016/j.fcl.2004.09.002.

[9] Lewis Gladius, Alva Preetam. Stress analysis of a flexible one-piece type first metatarsophalangeal joint implant. J Am Podiatr Med Assoc 1993;8(1):29-38, doi:http://dx.doi.org/10.7547/87507315-83-1-29.

[10] Flavin R, Halpin T, O’Sullivan R, FitzPatrick D, Ivankovic A, Stephens MM. A finite-element analysis study of the metatarsophalangeal joint of the hallux rigidus. J Bone Joint Surg Br 2008;90(10):1334-40, doi:http://dx.doi.org/ 10.1302/0301-620X.90B10.20506.

[11] Williams N, Penrose J, Hose D. Computer model analysis of the Swanson and Sutter metacarpophalangeal joint implants. J Hand Surg Br 2000;25(2):21220, doi:http://dx.doi.org/10.1054/jhsb.1999.0352.

[12] Mayich D Joshua, Novak Alison, Vena Daniel, Daniels Timothy R, Brodsky James W. Gait analysis in orthopedic foot and ankle surgery-topical review, part I: principles and uses of gait analysis. Foot Ankle Int 2014;35(1):80-90, doi: http://dx.doi.org/10.1177/1071100713508394.

13] Gefen A, Megido-Ravid M, Itzchak Y, Arcan M. Biomechanical analysis of the three-dimensional foot structure during gait: a basic tool for clinical applications. J Biomech Eng 2000;122:630-9, doi:http://dx.doi.org/10.1115/ 1.1318904

[14] Netter Frank H. Atlas de anatomía humana. 4ta edición Barcelona, España: Elsevier, Masson; 2007.

[15] Kapandji AI. Fisiología articular 2, miembro inferior. 5ta edición Editorial médica panamericana; 1998.

[16] Calvo Alberto, Viladot Ramón, Giné Josep, Alvarez Fernando. The importance of the length of the first metatarsal and the proximal phalanx of hallux in the etiopathogeny of the hallux rigidus. Foot Ankle Surg 2009;15(2):69-74, doi: http://dx.doi.org/10.1016/j.fas.2008.08.001.

[17] Clayton NLC, Andrew CK, Dean JS. Autogenous capsular interpositional arthroplasty surgery for painful hallux rigidus: assessing changes in range of motion and postoperative foot health. J Foot Ankle Surg 2015;54(1):29-36, doi:http://dx.doi.org/10.1053/j.jfas.2014.09.004.

[18] García A, Bayod J, Prados JC, Losa MA, Kevin T, Becerro de Bengoa R, et al. Finiteelement simulation of flexor digitorumlongus or flexor digitorumbrevis tendon transfer for the treatment of claw toe deformity. J Biomech 2009;42 (11):1697-704, doi:http://dx.doi.org/10.1016/j.jbiomech.2009.04.031.

[19] Bayod J, Becerro de Bengoa R, Losa M, Doblare M. Mechanical stress redistribution in the calcaneus after autologous bone harvesting. J Biomech 2012;45(7):1219-26, doi:http://dx.doi.org/10.1016/j.jbiomech.2012.01.043.

[20] Buddy DR, Hoffman AS, Schoen Frederick J, Lemons Jack E. Medical applications of silicones in biomaterials science. An introduction to materials in medicine. third edition Kidlingtone, Oxford, UK: Academic Press Elsevier: 2013 p. 1106 (Chapter II.5.18).

[21] Morales E, Bayod J, Becerro de Bengoa R, Losa M, Doblare M. Influence of first proximal phalanx geometry on hallux valgus deformity: a finite element analysis. Med Biol Eng Comput 2015;53(7):645-53, doi:http://dx.doi.org/ 10.1007/s11517-015-1260-4.

[22] MatWeb Material Property data copyright 1996-2016. Recuperado de: http:// asm.matweb.com/search/SpecificMaterial.asp?bassnum=MTP641.

[23] Bayod Javier, Losa Iglesias Marta, Becerro de Bengoa Vallejo Ricardo, Prados Frutos Juan Carlos, Jules Kevin T, Doblaré Manuel. Advantages and drawbacks of proximal interphalangeal joint fusion versus flexor tendon transfer in the correction of hammer and claw toe deformity a finite/element study. J Biomech Eng 2010;132(5):051002, doi:http://dx.doi.org/10.1115/1.4001115.

[24] Shin J, Yue N, Untaroiu CD. A finite element model of the foot and ankle for automotive impact applications. Ann Biomed Eng 2012;40(12):2519-31, doi: http://dx.doi.org/10.1007/s10439-012-0607-3.

[25] Harrison WJ, Loughead JM. Silastic metatarsophalangeal arthroplasty: very long-term results of single-stem implants in degenerative joint disease. Foot 2003;13:146-50, doi:http://dx.doi.org/10.1016/S0958-2592(03)00037-3.

[26] Bankes MJK, Shah RR, Grace DL. Swanson double-stem arthroplasty a survivorship analysis. Foot Ankle Surg 1999;5(4):235-43, doi:http://dx.doi. org/10.1046/j.1460-9584.1999.00176.x.

[27] Morgan S, Aaron Ng, Cloug T. The long-term outcome of silastic implant arthroplasty of the first metatarsophalangeal joint: a retrospective analysis of one hundred and eight feet. Int Orthop 2012;36(9):1865-9, doi:http://dx.doi. org/10.1007/s00264-012-1576-8.

[28] van Duijvenbode CD, Bulstran GH, Nijsse BA. Nineteen-years follow-upof the silastic double stemmed hinge prosthesis of the first metatarsophalangeal joint. Foot Ankle Surg 2013;19(1):27-30, doi:http://dx.doi.org/10.1016/j. fas.2012.08.001.

[29] Zeng Yan-Jun, Sun Xiao-peng, Yang Jian, Wu Wei-hua, Xu Xiao-hu, Yan Yi-ping. Mechanical properties of nasal fascia and periosteum. Clin Biomech (Bristol, Avon) 2003;18(8):760-4, doi:http://dx.doi.org/10.1016/S0268-0033(03) 00136-0.

[30] Trabelsi Nir, Milgrom Charles, Yosibash Zohar. Patient-specific FE analyses of metatarsal bones with inhomogeneous isotropic material properties. J Mech Behav Biomed Mater 2014;29:177-89, doi:http://dx.doi.org/10.1016/j. jmbbm.2013.08.030.

[31] Kanzaki Noriyuki, Nishiyama Takayuki, Fujishiro Takaaki, Hayash Shinya, Hashimoto Shingo, Kuroda Ryosuke, et al. Flexible hinge silicone implant with or without titanium grommets for arthroplasty of the first metatarsophalangeal joint. J Orthop Surg (Hong Kong) 2014;22(1):42-5. https://www.ncbi. nlm.nih.gov/pubmed/24781612.

[32] Granberry WM, Noble PC, Bishop JO, Tullos HS. Use of a hinged silicone prosthesis for replacement arthroplasty of the first metatarsophalangeal joint J Bone Jt Surg Am 1991;73(10):1453-9. https://www.ncbi.nlm.nih.gov/ pubmed/1748694.

[33] Joyce TJ, Unsworth A. A literature review of failures of the Swanson finger prosthesis in the metacarpophalangeal joint. Hand Surg 2002;7(1):139-46. 